\title{
LISA and its In-Flight Test Precursor SMART-2
}

S. Vitale, P. Bender ${ }^{b}$, A. Brillet, S. Buchman ${ }^{\text {d }}$, A. Cavalleri', M. Cerdonio ${ }^{\text {e }}$, M. Cruise, C. Cutler, K. Danzmann $^{\text {, }}$ R. Dolesia , W. Folkner', A. Gianolio', Y. Jafry', G. Hasinger', G. Heinzel ${ }^{\text {b }}$, C. Hogan', M. Huellera,

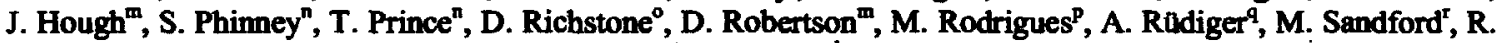
Schilling', D. Shoemaker', B. Schutz', R. Stebbins', C. Stubbs', T. Sumner ${ }^{\mathrm{n}}$, K. Thome", M. Tinto', P. Touboulp, H. Ward ${ }^{\mathrm{m}}$, W. Weber and W. Winkler ${ }^{\mathrm{q}}$

a Dipartimento di Fisica, Universita di Trento I-38050, Povo, Trento, Italy

b Joint Institutes for Laboratory Astrophysics, Boulder, Colorado, USA.

- Observatoire de la Côte d'Azur, Nice, France

d W.W. Hansen Experimental Physics Labs, Stanford University, Stanford, California

- Dipartimento di Fisica Universita di Padova, Italy

f Department of Physics \& Astronomy, University of Birmingham, UK

g Max-Planck-Institut für Gravitationsphysik (Albert-Einstein-Institut) Golm, Germany

h Max-Planck-Institut fưr Gravitationsphysik (Albert-Einstein-Institut) and Universităt Hannover, Hannover, Germany

Jet Propulsion Laboratory, Pasadena, California,USA

ESA-ESTEC, Nordwijk, The Netherlands

Max-Planck-Institurt für Extraterrestrische Physik, Garching, Germany

Departments of Physics and Astronomy, University of Washington, Seattle, Washington, USA

Department of Physics and Astronomy, University of Glasgow, Glasgow, UK

California Institute of Technology, Pasadena, Califomia, USA

Department of Astronomy, University of Michigan, Ann Arbor, Michigan, USA

ONERA, Chatillon, France

Max-Planck-Institut für Quantenoptik, Garching, Germany

Rutherford Appleton Laboratory, Chilton-Didcot, UK

Massachusetts Institute of Technology, Cambridge, Massachusetts, USA

NASA Goddard Space Flight Center, Greenbelt, Maryland, USA

The Blackett Laboratory, Imperial College of Science, Technology \& Medicine, London, UK

LISA will be the first space-borne gravitational wave observatory. It aims to detect gravitational waves in the $0.1 \mathrm{mHz}+1 \mathrm{~Hz}$ range from sources including galactic binaries, super-massive black-hole binaries, capture of objects by super-massive blackholes and stochastic background. LISA is an ESA approved Cornerstone Mission forescen as a joint ESA-NASA endeavour to be launched in 2010-11. The principle of operation of LISA is based on laser ranging of test-masses under pure geodesic motion. Achieving pure geodesic motion at the level requested for LISA, $3 \times 10^{-15} \mathrm{~ms}^{-2} / \sqrt{\mathrm{Hz}}$ at $0.1 \mathrm{mHz}$, is considered a challenging technological objective. To recuce the risk, both ESA and NASA are pursuing an in-flight test of the relevant technology. The goal of the test is to demonstrate geodetic motion within one order of magnitude from the LISA performance. ESA has given this test as the primary goal of its technology dedicated mission SMART-2 with a launch in 2006. This paper describes the basics of LISA, its key technologies, and its in-flight precursor test on SMART-2.

\section{INTRODUCTION}

LISA [1-2] will be the first high sensitivity spaceborne gravitational wave detector. LISA consists of a constellation of 3 satellites in heliocentric orbits. The orbits are adjusted such that the three spacecraft maintain an equilateral triangle formation with a
$5 \times 10^{6} \mathrm{~km}$ side (Figure 1). Each spacecraft (Figure 2) contains a pair of test-masses, of approximately $1 \mathrm{~kg}$, nominally in pure geodesic motion. Each test mass is the end-mirror of a single arm interferometer, the other end-mirror being in one of the other two spacecraft. Two semi-independent two-arm interferometers can then be formed by 


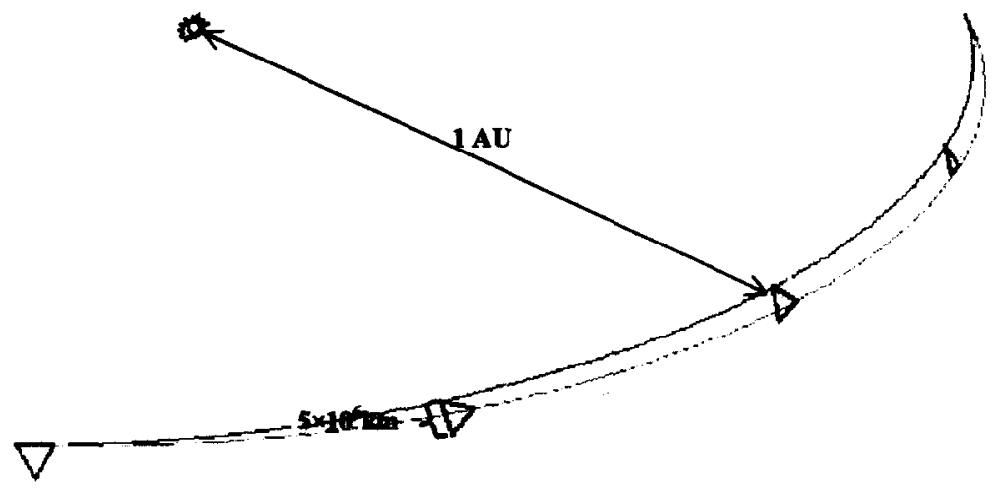

Figure 1. LISA orbits. The three spacecraft follow independent heliocentric orbits with parameters adjusted such that the distances among the spacecraft are kept constants to within $\approx 1 \%$ at $5 \times 10^{6} \mathrm{~km}$. The rotating triangle represents the laser beams joining the spacecraft located at the vertices. The drawing is approximately to scale. The Earth (not represented) leads the formation by $20^{\circ}$.

taking the difference of the signals from the 3 independent arms. LISA strain sensitivity goal is $\mathrm{S}_{\mathrm{h}} \approx 4 \times 10^{-21} 1 / \sqrt{\mathrm{Hz}}$ at around $3 \mathrm{mHz}$.

The anticipated bandwidth is between $0.1 \mathrm{mHz}$ and $1 \mathrm{~Hz}$, with the noise increasing at both ends of the band (Figure 3).

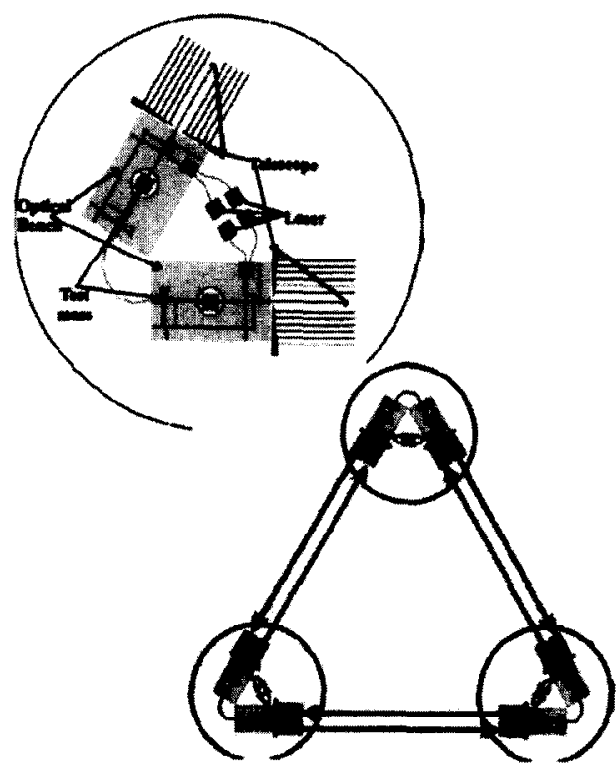

Figure 2. Schematic of LISA layout. Top: layout within each spacecraft. Bottom: LISA constellation layout showing the six independent laser beams exchanged among the spacecraft.
LISA candidate sources have been discussed in detail in Ref 1 and reviewed more recently in Ref 3. A non-exhaustive list is indicated in Table 1.

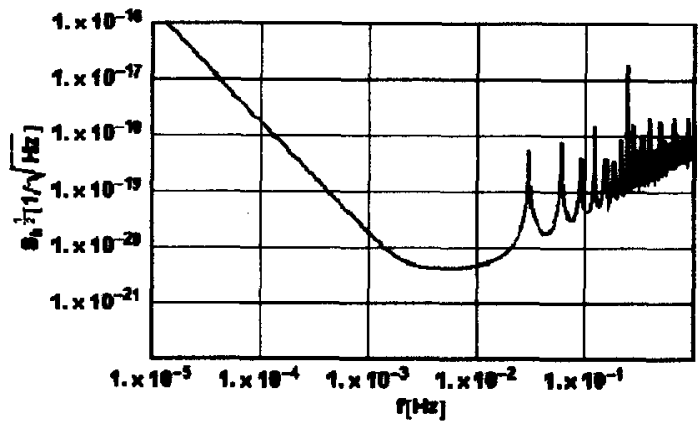

Figure 3. LISA estimated strain noise spectrum for an optimally oriented and polarized gravitational wave. The pattern of narrow insensitive bands at high frequency comes from the finite light travel time and washes out nearly completely for any source direction because of the anterna rotation when averaged over a year.

\section{LISA TECHNOLOGY}

\section{LISA hardware key elements [4] are:}

- Laser metrology with a resolution of $40 \mathrm{pm} / \sqrt{\mathrm{Hz}}$ for $f>3 \mathrm{mHz}$ on the round trip optical path difference between two adjacent interferometer arms. 
Table 1

LISA Sources

\begin{tabular}{|c|c|c|c|}
\hline Source & $\begin{array}{l}\text { Frequency } \\
(\mathrm{mHz})\end{array}$ & $\begin{array}{l}\text { Expected } \\
\text { SNR }\end{array}$ & Remarks \\
\hline \multicolumn{4}{|l|}{ Identified Galactic Binaries } \\
\hline $\begin{array}{l}\text { (known period, position, mass, plus } \\
\text { distance within factor 2) }\end{array}$ & $0.15-3.5$ & $10-700$ & At least 16 identified sources \\
\hline Other Galactic Binaries & $0.1-10$ & $>\mathbf{2 0}$ & $\begin{array}{c}\text { For resolvable binaries above the } \\
\text { galactic background }\end{array}$ \\
\hline Super-Massive Black-Hole Mergers & $0.1-1000$ & $30-10^{4}$ & $\begin{array}{c}\text { Detectable for } 10^{3} \mathrm{M}_{\odot}<\mathrm{M}<10^{7} \mathrm{M}_{\odot} \\
\text { and } \mathrm{z}<10\end{array}$ \\
\hline $\begin{array}{l}\text { Gravitational capture by a super- } \\
\text { massive black-hole }\end{array}$ & $1-100$ & $>10$ & \\
\hline Stochastic background & - & - & $\begin{array}{l}\text { Sagnac combination of beams allows } \\
\text { subtraction of instrument noise }\end{array}$ \\
\hline
\end{tabular}

Interferometers are not based on simple reflection on the end mimors as, due to the large travel distance, the collected power would be insufficient. Instead, a new laser signal, phaselocked to the incoming beam, is generated at the receiving spacecraft and sent back to the first.

In this way, 6 independent phase signals, one for each beam, are available and may be combined in different ways, an important feature for signal extraction. For instance, they may be combined to form the symmetric Sagnac observable, which has a greatly reduced sensitivity to gravitational waves and allows for measurement of most instrument noise sources [5].

Laser metrology is composed in turn by a few essential elements.

- 12× Nd:Yag, $1.064 \mu \mathrm{m}$ ring-laser of approximately $1 \mathrm{~W}$ power constitute the light source for the metrology. 4 laser heads are hosted on board each spacecraft for redundancy.

- 6XULE optical benches (2 per spacecraft) carrying all the necessary optical components

- $6 \times 30 \mathrm{~cm}$, Dall-Kirkham telescopes for collecting and sending the beams

- An Ultra-Stable Oscillator (USO) is used as a local clock for the complex beat-note processing. As a sufficiently stable USO is as yet unavailable, the signal from a master USO is circulated as a modulation of the laser beam to achieve synchronisation of the local oscillators on board the three spacecraft.

- A 19 degrees of freedom per spacecraft control system whose main purposes are to minimize the disturbances on the test-masses and to keep the 6 laser beams aligned and locked. The main constituents of this control systems are:

- Motion sensors for the six degrees of freedom of each test-mass relative to its host spacecraft. Each of these so-called gravitational sensors [6-7] is comprised of a set of capacitive, parametric bridges reading the capacitances between the test-mass and a series of properly shaped electrodes.

- A set of micro-Newton level Field Emission Electric Propulsion (FEEP)[8] thrusters that may exert forces and torques on the spacecraft along its 6 degrees of freedom.

The thrusters system is driven by the signals coming from the gravitational sensors. The control loop tries to minimize the displacement of each test-mass, along the axis defined by the laser beam impinging on it, measured relative to a reference point fixed to the spacecraft. As 
these axes are not degenerate but lie 60 degrees apart, the spacecraft motion is fully controlled in the plane $(x-y)$ containing both beams. The residual displacement is expected to be $<2.5$ $\mathrm{nm} / \sqrt{\mathrm{Hz}}$, in the measuring bandwidth for a thrust noise of $\approx 0.1 \mu \mathrm{N} / \sqrt{\mathrm{Hz}}$. The spacecraft is also controlled along $z$ using the gravitational sensors signals to a slightly more relaxed figure. In attitude the spacecraft is kept aligned with the wave-fronts of the beams received from both distant spacecraft. A control to better than 8 $\mathrm{nrad} / \sqrt{\mathrm{Hz}}$ is expected.

As the spacecraft triangle formation will "breath" in angle by $\approx 0.6^{\circ}$ over the year, the angles between the telescope must also be controlled. This is achieved by an articulation actuated by a precision actuator.

Along the degrees of freedom not aligned with the laser beam axes, the test-masses are kept centred relative to their electrode housings by means of a force control loop. The force is due to electric fields generated by the electrodes. The force control loop is based on amplitude modulation of an ac carrier to avoid dc fields that are sources of a variety of stray force effects. The control loop gain is engineered to provide strong control at very low frequencies, but with a minimal spacecraft - mass coupling in the measurement bandwidth.

\section{GEODESIC MOTION AND LISA PERFORMANCE}

The sensitivity performance in Figure 3 is limited at low frequency by stray forces perturbing the masses out of their geodetics. Indeed, in the long wavelength limit for the gravitational signal, and for small signals, the separation $\Delta x$ between the two end-mirror masses, of mass $m$, belonging to one interferometer arm obeys an equation of motion:

$m \frac{d^{2} \Delta x}{d t^{2}}=\Delta F_{x}+m L \frac{d^{2} h}{d t^{2}}$

where $h(t)$ is the gravitational wave strain signal and $L$ is the umperturbed value of the test-masses separation. $\Delta \mathrm{F}_{\mathrm{x}}$ is the differential force either of nongravitational origin or due to local sources of gravitational field.
When converted to the frequency domain, eq. 1 shows that any force noise with spectral density $S_{\Delta r}$ would mimic a gravitational wave noise with density

$$
S_{h}^{1 / 2}=\frac{S_{\Delta \mathrm{F}}^{1 / 2}}{m L \omega^{2}}
$$
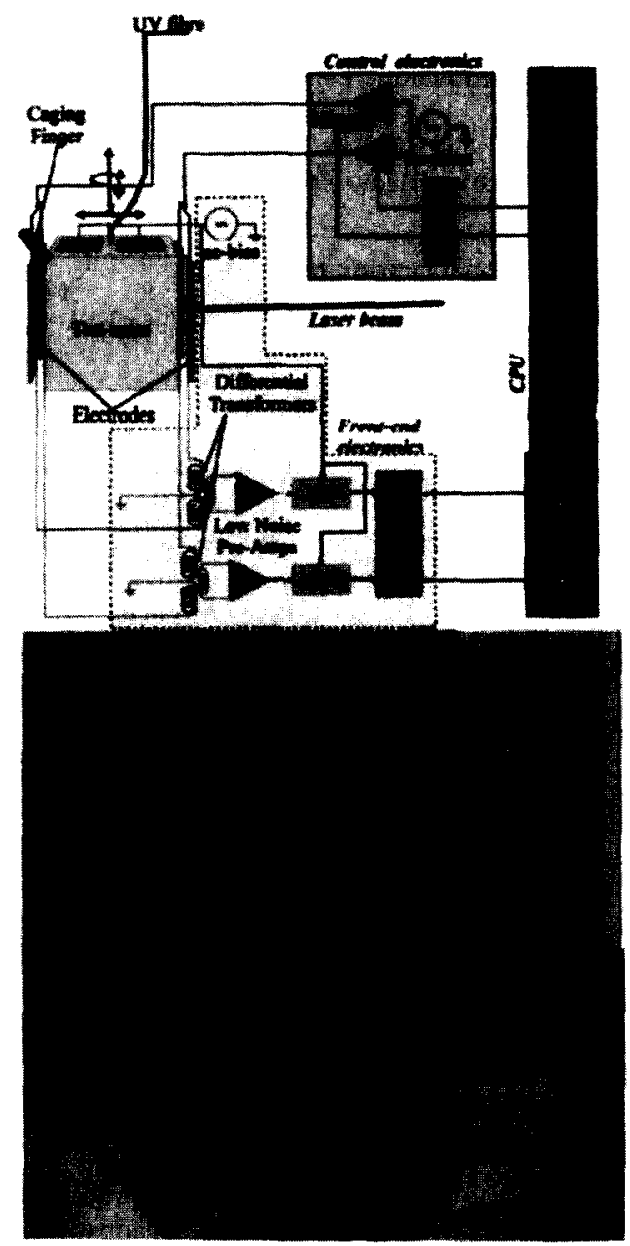

Figure 4. Top: block scheme of the gravitational sensor showing the sensing electrodes, the front-end electronics and the actuation electronics for 1 translation and one rotation degree of freedom. Bottom: a prototype of the gravitational sensor showing all electrodes.

where $\omega=2 \pi f$ and $f$ is the frequency of the measurement. 
As the effect decays with $\mathrm{f}^{2}$, above $\approx 3 \mathrm{mHz}$ the performance of LISA is expected to be set by the nearly shot noise limited displacement sensitivity of the laser interferometer of $\approx \mathbf{4 0} \mathrm{pm} / \mathrm{VHz}$.

This is achieved only if stray forces have a spectral density less than:

$$
\frac{S_{\Delta P}^{1 / 2}}{m}=3 \times 10^{-15}\left[1+\left(\frac{f}{3 m H z}\right)^{2}\right] \frac{m}{s^{2} \sqrt{H z}}
$$

down to a frequency $\mathrm{f}=0.1 \mathrm{mHz}$.

This is one of the critical goals for LISA. Unfortumately, in contrast to the other critical items, the achievement of this level of force isolation cannot be tested on ground.

One can calculate that, in the case of a single-mass/single-axis control loop, the residual acceleration of the test-mass is

$a=g+a_{n}=g+\underbrace{\left(x_{n}+\frac{F_{S / C}}{M \omega_{f b}^{2}}\right)}_{x_{n d}} \omega_{p}^{2}+\frac{f_{T / M}}{m}$

Here $g$ is the gravitational acceleration of the testmass, $m$ and $M$ are the mass values of the test-mass and of the spacecraft respectively. The disturbing forces that contribute to the stray acceleration $a_{n}$ in eq. (4) are:

- Those applied to the spacecraft $F_{S K C}$, including the thruster noise and difference in the gravitational acceleration between the test-mass and the spacecraft centre of mass.

- The forces acting on the test-mass, including, for instance, those due to thermal noise and pressure fluctuations.

- The contribution due to the sensor noise $x_{n}$ which drives the thrusters and produces a random force.

The residual coupling of the test-mass to the spacecraft is summarised by $\omega_{p}^{2}$, defined as $\mathrm{df}_{\mathrm{in}} / \mathrm{dx}=-\mathrm{m} \omega_{\mathrm{p}}^{2}$, with $\mathrm{f}_{\mathrm{imt}}$ the force that the spacecraft exerts onto the test-mass. A figure of $\left|\omega_{\mathrm{p}}^{2}\right| \leq 5 \times 10^{-7} \mathrm{~s}^{-2}$ is foreseen as a target. The gain of the control loop (applied force/displacement) divided by the spacecraft mass is instead represented by $\omega_{\mathrm{fb}}^{2}$. Neither $\omega_{\mathrm{p}}^{2}$ nor $\omega_{\mathrm{fb}}^{2}$ need to be real positive numbers. Because of the coupling $\omega_{p}^{2}$, to minimize $a_{n}$ it is important also to minimize the total residual jitter, $x_{\text {reb }}$ in the position of the test mass relative to the spacecraft, at least along the two directions of the laser beams, which is achieved by the "drag-free" control loop described above. $x_{\text {rel }}$ should have a power spectral density of $2.5 \mathrm{~nm} / \mathrm{VHz}$.

\section{SMART-2 AND THE TEST OF GLODESIC MOTION}

Very early during the various studies for LISA, the need for a technology demonstration mission was recognised. The first definite proposal for such a mission had been studied by the scientific commumity in 1998 and was known as ELITE [9]. That concept has now been widely recognized as a sufficient demonstration of the key technological aspects of LISA, and is now planned by ESA for flying, as the LISA Technology Package (LTP) (Figure 5), on board SMART-2 in 2006 as an ESA mission. A similar package, known as the disturbance reduction system (DRS), is being studied by NASA and may fly on SMART-2 also.

The basic idea behind the LTP is to squeeze one LISA arm from $510^{6} \mathrm{~km}$ to a few centimetres and place it aboard a single $S / C$.

This scheme is an almost ideal Einstein "geodesic" deviation experiment with a negligible separation between the two test-particles that suppresses the sensitivity to large scale curvature.

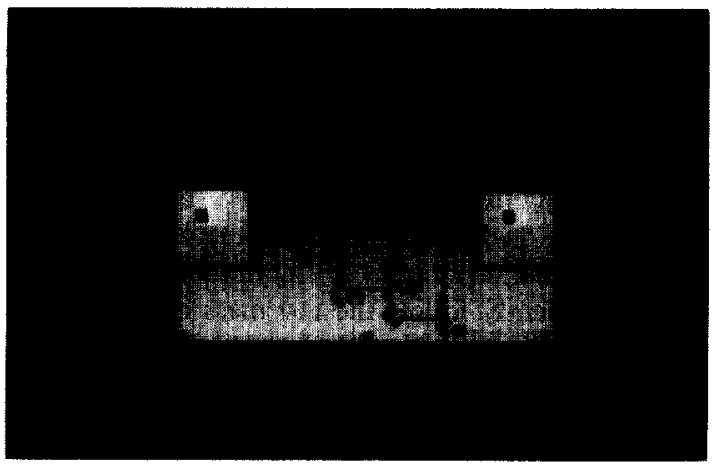

Figure 5. Concept of the Lisa Technology Package: two gravitational sensors (a) and a laser interferometer mounted on an ultra-stable optical bench (b). The laser source (c) and the front-end electronics are located outside the main, ultra-stable box.

It is accepted that, in order for the test to be considered significant, it must verify the ability to 
set a test-mass in purely gravitational free-fall within one order of magnitude from the LISA goal both in amplitude and in frequency. The test goal is then to demonstrate:

$S_{1}^{1 / 3}(f) \leq 3 \times 10^{-14}\left[1+\left(\frac{f}{3 \mathrm{mHz}}\right)^{2}\right] \frac{\mathrm{m}}{\mathrm{s}^{2}} \frac{1}{\sqrt{\mathrm{Hz}}}$

for frequencies

$1 \mathrm{mHz} \leq \mathrm{f} \leq 30 \mathrm{mHz}$

In the LTP, the tests masses are surrounded by their position sensing electrodes and constitute two gravitational sensors nominally identical to those of LISA. The gravitational sensors drive a drag-free control loop in close analogy to LISA.

A laser interferometer (Figure 5) gives an independent reading of the relative position of the test-masses along their separation line that coincides with the direction of the laser beam. The laser readout is affected by a noise $x_{n \text {,mer }}$ The requested performance (see below) is shown in Figure 6.

There is an important point where the analogy between LISA and the LTP breaks down. In LISA each spacecraft hosts two test-masses as for SMART2. However these two test-masses belong to different interferometer arms. This has an important consequence on the spacecraft control. For LISA, the control logic simultaneously centres the spacecraft relative to both test-masses along the axes defined by the incoming laser beams, a "two mass/two axes" scheme that is possible only if the two axes are not aligned. Though the two axes per LISA spacecraft are not orthogonal, in which case the scheme would be self-explanatory, their 60 degree angular separation still permits the spacecraft to independently follow the test mass along the two laser measurement axes.

On SMART2 instead, in order to be able to measure differential acceleration, the sensitive axes of the two test-masses have to be aligned (Figure 5). This forces one to develop a force control loop that actively drags at least one of the test-masses along with the spacecraft also along the measurement axis. The control loop has to insure large gain below the measuring bandwidth and the minimum possible gain within the bandwidth. For this reason it usually called a low frequency suspension.

If indeed the spacecraft follows one of the testmasses, while the other is carried along by a force loop, the laser interferometer will generate a displacement signal:

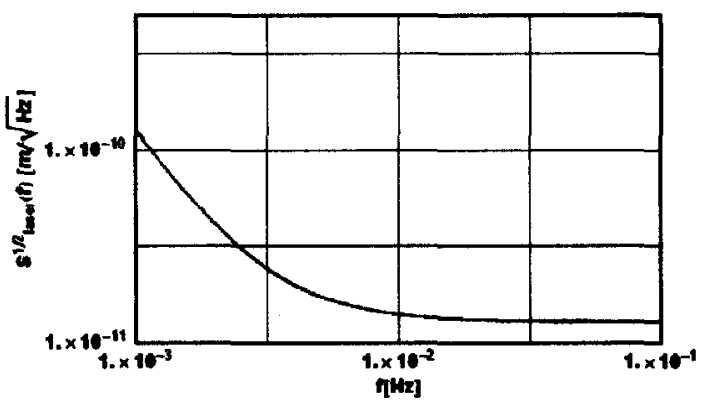

Figure 6. Laser interferometer performance requirement.

$$
\begin{aligned}
& \delta \mathrm{x}_{\text {lesser }} \approx \frac{1}{\omega^{2}-\left(\omega_{1 \mathrm{~B}}^{2}+\omega_{\mathrm{p} 2}^{2}\right)} \times[ \\
& \left(\omega_{1 \mathrm{E}}^{2}+\omega_{\mathrm{p} 2}^{2}-\omega^{2}\right) \mathrm{x}_{\mathrm{n}, \max }+\delta \mathrm{x}\left(\omega_{\mathrm{ti}}^{2}+\omega_{\mathrm{p} 2}^{2}\right)+\mathrm{x}_{\mathrm{n} 2} \omega_{1 \mathrm{~B}}^{2} \\
& -[\left(\omega_{1 \mathrm{f}}^{2}+\omega_{\mathrm{p} 2}^{2}\right)-\underbrace{\left.\left.-\omega_{\mathrm{pl}}^{2}\right]\right]\left(\mathrm{x}_{\mathrm{n} 1}+\frac{\mathrm{F}_{\mathrm{s} / \mathrm{C}}}{M \omega_{\mathrm{n}}^{2}}\right)+\frac{f_{T / M 1}}{m}}_{\mathrm{a}_{\mathrm{n}}} \\
& \left.-\frac{f_{T / M 2}}{m}\right]
\end{aligned}
$$

In eq. (7) $\omega_{1 \mathrm{f}}^{2}$ is the gain of the low frequency suspension, the numbers 1 or 2 label the test-masses, and finally $\delta x$ is the distortion of the baseline separating the two gravitational sensors.

By comparing eq. (4) with eq. (7) one can draw a few simple conclusions. The way the test is performed clearly adds a few extra noise sources. These sources may add to the one to be measured, marked as $a_{n}$ in eq. (7), but may even subtract from them.

For instance, the laser noise, or the contribution of the baseline distortion, incoherently add to $a_{n}$ and must thus simply be kept fow. This is the origin of the requirement on the laser noise in Figure 6. The baseline distortion is kept low by the ultra-stable optical bench, which acts as a mechanical reference for the assembly, and by the $0.1 \mathrm{mK} / \mathrm{VHz}$ thermal stability in the relevant bandwidth.

The contribution of the noise of the gravitational sensor $n$. 2, cannot be made much smaller than the 
rest, and its effect must be taken into accoumt in the final performance estimate. An alternative scheme, where the second test-mass is set free for some limited time with no force control applied, is also being considered to suppress this sounce. However the comparatively large expected drift rate seems to make the scheme marginal.

A coherent additional force source is represented by the term $-\left(\omega_{1 \mathrm{a}}^{2}+\omega_{\mathrm{p} 2}^{2}\right)\left(\mathrm{x}_{\mathrm{n} 1}+\mathrm{F}_{\mathrm{s} / \mathrm{c}} / \mathrm{M} \omega_{\mathrm{fb}}^{2}\right)$ in eq. (7). If, for instance, $\omega_{1 \mathrm{f}}^{2}+\omega_{\mathrm{p} 2}^{2}=\omega_{\mathrm{pl}}^{2}$, the contribution of $x_{\text {rel }}$ to the noise would be cancelled and the overall noise would be underestimated. The instrument would indeed act as an ideally matched gravity gradiometer, insensitive to the platform motion.

$\omega_{\mathrm{p} 1}^{2}$ and $\omega_{\mathrm{p} 2}^{2}$ cannot be chosen at will, and thus a careful design should minimize their values. Because the gravitational sensors are nominally identical, $\omega_{\mathrm{p} 2}^{2} \approx \omega_{\mathrm{p} 1}^{2}$. In addition they are both expected to be negative $\left(\omega_{\mathrm{p} 2}^{2}, \omega_{\mathrm{p} 1}^{2}<0\right)$, making the test-masses, in the absence of the drag-free and low frequency suspension control loops, unstable. Fortunately, in order to stabilize the test-mass, an optimised low frequency suspension 7 provides a gain $\omega_{1 \mathrm{f}}^{2} \approx-2 \omega_{\mathrm{p} 2}^{2}$, such that $\omega_{1 \mathrm{~s}}^{2}+\omega_{\mathrm{p} 2}^{2} \approx-\omega_{\mathrm{pl}}^{2}>0$. It then follows that:

$$
\begin{aligned}
& {\left[\omega_{\mathrm{p} 1}^{2}-\left(\omega_{\mathrm{fs}}^{2}+\omega_{\mathrm{p} 2}^{2}\right)\right]\left(\mathrm{x}_{\mathrm{n} 1}+\mathrm{F}_{\mathrm{s} / \mathrm{c}} / \mathrm{M} \omega_{\mathrm{fb}}^{2}\right) \approx} \\
& \approx 2 \omega_{\mathrm{p} 1}^{2}\left(\mathrm{x}_{\mathrm{n} 1}+\mathrm{F}_{\mathrm{s} / \mathrm{c}} / \mathrm{M} \omega_{\mathrm{fb}}^{2}\right)
\end{aligned}
$$

Also the force $f_{T / M 2}$ may subtract coherently from

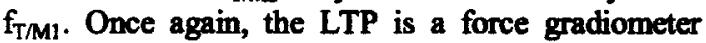
and cannot measure any force identically applied to both test-masses. This is an intrinsic limitation of the test. However the lesser known and more worrisome disturbances, like the Brownian motion of the test-mass due to dielectric losses, the radiation pressure differences across the test-mass, the radiometer effect, and the stray residual dc-fields, are indeed independently applied to the two sensors and will be detected in the test.

\section{CONCLUDING REMARKS}

By the time LISA will be launched, it is likely that second generation ground-based gravitational wave detectors will be operating and detecting signals from stellar-mass black-hole mergers at frequencies in the range $10-1000 \mathrm{~Hz}$. Increasing confidence in data analysis tools and in the physical characterisation of detector noise and sensitivity may have progressively changed early detections into ongoing astronomical observations.

LISA will open 4 more frequency decades of the spectrum of the newly born gravitational wave astronomy with a few bright standard candles to validate its observations. In addition some of the expected signals will give the unique possibility to perform observations in the strong gravitational field limit. This is the case both for super-massive blackboles mergers and for the gravitational capture of stellar mass objects by a super-massive black bole. This last case would even give a test of the "mo-hair" theorem. ${ }^{3}$

LISA related technology is rapidly reaching maturity. The space accelerometers and ground based interferometers constitute the obvious heritage upon which this technology development builds. A comprehensive technology development plan is shaping up both in Europe and in the USA, with some items already being studied with the aim of developing engineering models in preparation for SMART-2 in 2006.

A key part of this technological effort is to devise a ground-based test and validation plan to reduce the risk of the in-flight test. Though a full test of free-fall at the level requested by eq. 5 does not seem possible for a $1 \mathrm{~kg}$ size test-mass, torsion pendulums have been shown10 to have sensitivities of order $5 \times 10^{-13} \mathrm{~N} / \mathrm{HHz}$ at frequencies even below 1 $\mathrm{mHz}$ for bodies of shapes similar to that of the LISA test-mass. This motivates the current effort to develop sensitive torsion pendulum test-benches for the test-mass [7], in order to put an upper limit to a large class of the most worrisome disturbances not far from the in-flight test goal.

LISA would then take advantage of a risk reduction route comprised of one order of magnitude performance leaps from ground bases test-benches to the in-flight test and then to the final mission.

\section{REFLRENCES}

1 P. Bender et al. "LISA Laser Interferometer Space Antenna: A Cornerstone Mission for the Observation of Gravitational Waves. System and Technology Study Report" ESA-SCI(2000)1 1, (Jume 2000) 
2 A. Hammesfahr, Classical and Quantum Gravity 18,4045 (2001).

3 “LISA Science Requirements” E.S. Phinney et al. Presentation to the LISA International Science Team, 12 December 2001, Trento, Italy.

4 ESTEC contract 13631/88/NL/MS, LISA study of the Laser Interferometer Space Antenna, Final Report. (Astrium 2000)

5 F. B. Estabrook, M. Tinto, and J. W. Armstrong, Phys. Rev. D62, 042002 (2000). M. Tinto, J. W. Armstrong, and F.B. Estabrook, Classical and Quantum Gravity 18, 4081 (2001).

6 P. Touboul, M. Rodrigues, and G. M. Le Clerc, Classical and Quantum Gravity 13 A259, 1996.

7 A. Cavalleri, R. Dolesi, G. Fontana, M. Hueller, J. Turneaure, S. Vitale and W. Weber, Classical and Quantum Gravity 18, 4133 (2001).

8 C. Bartoli, H. von Rhoden, S. Thompson, and J. Blommers, J. Phys. D: Appl. Phys. 17, 2473 (1984)

9 ELITE, European LISA Technology Demonstration Satellite, Proposal May 1998.(Unpublished)

10 Y. Su et al., Phys. Rev. D50, 3614 (1994) 